\title{
True bugs (Hemiptera, Heteroptera) as psyllid predators (Hemiptera, Psylloidea)
}

\author{
Dušanka Jerinić-Prodanović', Ljiljana Protić \\ I University of Belgrade, Faculty of Agriculture, Nemanjina 6, 11080 Zemun, Serbia 2 Natural History Mu- \\ seum, Njegoševa 51, 11000 Belgrade, Serbia \\ Corresponding author: Dušanka Jerinić-Prodanović (dusanka@agrif.bg.ac.rs)
}

Academic editor: A. Popov | Received 14 November 2012 | Accepted 28 May 2013 | Published 30 July 2013

Citation: Jerinić-Prodanović D, Protić L (2013) True bugs (Hemiptera, Heteroptera) as psyllid predators (Hemiptera, Psylloidea). In: Popov A, Grozeva S, Simov N, Tasheva E (Eds) Advances in Hemipterology. ZooKeys 319: 169-189. doi: $10.3897 /$ zookeys.319.4316

\begin{abstract}
Data on natural enemies of psyllids are rare and can usually be found in papers about economically significant species. During an investigation of psyllid fauna in Serbia, natural enemies were investigated, too. True bugs were the most numerous among them. From 28 psyllid species, 21 species of true bugs from families Anthocoridae and Miridae were reared. Seven species of Anthocoridae were identified: Anthocoris amplicollis (Horváth, 1839), A. confusus Reuter, 1884, A. nemoralis (Fabricius, 1794), A. nemorum (Linnaeus, 1761), Orius majusculus Reuter, 1884, O. minutus (Linnaeus, 1758) and O. niger Wolff, 1811. The following 14 species of Miridae were identified: Atractotomus mali Meyer-Dür, 1843, Campylomma verbasci (Meyer-Dür, 1843), Deraeocoris flavilinea (A. Costa, 1862), D. ruber (Linnaeus, 1758), D. lutescens (Schilling, 1836), Heterocordylus genistae (Scopoli, 1763), Hypseloecus visci (Puton, 1888), Malacocoris chlorizans Panzer, 1794, Miris striatus (Linnaeus, 1758), Orthotylus marginalis Reuter, 1884, Psallus assimilis Stichel, 1956, Ps. quercus Kirschbaum, 1856, Ps. flavellus Stichel, 1933 and Pseudoloxops coccinea (Meyer-Dür, 1843). The aim of the research was to provide list of true bugs recorded as predators of psyllids in order to preserve their diversity and significance, especially on cultivated plants.
\end{abstract}

\section{Keywords}

Psylloidea, Heteroptera, predators, natural enemies, Serbia 


\section{Introduction}

Predators of psyllids (Psylloidea) have been poorly known. So far, detailed researches were carried out only on the predators of economically significant species, such as pear psyllids Cacopsylla pyri (Linnaeus, 1758), C. pyricola (Foerster, 1848) and C. pyrisuga (Foerster, 1848); apple psyllid C. mali (Schmidberger, 1836) and eucalyptus psyllids from the subfamily Spondyliaspidinae (Jonsson 1983, Herard 1985, 1986, Santas 1987, Erler 2004, Horton et al. 2004, Sigsgaard et al. 2006, Jauset et al. 2006, Luiz de Queiroz et al. 2012). There are too few data on predators of other psyllid species. Hodkinson and Flint (1971) investigated predators of ash psyllid, Psyllopsis fraxini (Linnaeus, 1758), in England, and Harizanova et al. (2012), predatory complex of Acizzia jamatonica (Kuwayama, 1908) in Bulgaria. In these papers, the most represented are psyllid predators from the order Hemiptera (suborder Heteroptera) followed by Coleoptera, Neuroptera, Diptera, Dermaptera and Acari. Within the Heteroptera, the most numerous in species families are Anthocoridae, Miridae and Nabidae. A polyphagous species, Anthocoris nemoralis (Fabricius, 1794), was most frequently found, with a preference for the species from superfamily Psylloidea (Jonsson 1983, Herard 1986). Anthocoris nemoralis (Anthocoridae) was introduced from Europe to North America (British Columbia) in 1963 in order to control C. pyricola, where its establishment was successful. Besides giving satisfactory effects, this species also spread in the new environment suppressing autochthonous species Anthocoris antevolens White, 1879 and $A$. melanocerus Reuter, 1884, which are most common anthocorid predators in orchards (Herard 1986, Horton et al. 2004).

Data on psyllid predators in Serbia relate only to the predators of pear psyllids (Pavićević 1977, Grbić et al. 1989, Jerinić-Prodanović et al. 2010).

Pavićević (1977) found a large number of predatory species, among which two were from family Anthocoridae. Grbić et al. (1989) recorded four species of Heteroptera: Anthocoris nemoralis and Orius sp. (both Anthocoridae), Pilophorus clavatus (Linnaeus, 1767) (Miridae) and Nabis pseudoferus Remane, 1949 (Nabidae), while JerinićProdanović et al. (2010) reported seven species: Anthocoris nemoralis (Fabricius, 1794), A. nemorum (Linnaeus, 1761), Orius (Heterorius) minutus (Linnaeus, 1758) and Orius (Orius) niger Wolff, 1811 from the family Anthocoridae and Campylomma verbasci (Meyer-Dür, 1843), Deraeocoris (Deraeocoris) ruber (Linnaeus, 1758) and Deraeocoris (Knightocapsus) lutescens (Schilling, 1836) from the family Miridae.

There is no data on other predatory psyllid species in Serbia.

\section{Methods}

Insect material was collected from 419 localities within the whole territory of the Republic of Serbia. Investigations were carried out in the period from 2005 to 2010, in field conditions and in the laboratory of the Faculty of Agriculture in Zemun, University of Belgrade. Locality mapping was carried out in World UTM (Universal Trans- 
verse Mercator) cartographic projection. Determination of coordinates of investigated localities in the field was carried out using GPS devices Geoexplorer 3 (Trimble) and E-trex Vista Hcx (Garmin), with an accuracy of 3 to 5 meters.

Adults of predatory true bugs were collected from psyllid colonies by an aspirator and their larvae were collected together with plant material and psyllids and further reared to adults in laboratory conditions in Petri dishes.

The species identification of Heteroptera was based on Wagner (1970-1971, 1975), Péricart (1972) and Kerzhner and Josifov (1999).

A part of the material is deposited in the first author's collection in the Faculty of Agriculture, University of Belgrade, and another part, in the second author's collection in Natural History Museum, Belgrade.

\section{Results and discussion}

We collected and reared 21 true bug species predating on 28 psyllid species (Table 1) from 44 localities (Fig. 1 and Table 2). The identified true bugs belong to families Anthocoridae and Miridae.

\section{Anthocoridae}

\section{1) Anthocoris amplicollis (Horváth, 1839)}

Trophic status. Zoophagous.

Distribution. Europe.

Prey. Psylla buxi (Linnaeus, 1758), from Buxus sempervirens, Nova Galenika,

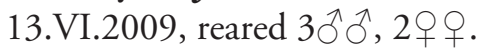

A. amplicollis was already reported in Serbia (Protić and Stojanović 2003) but the above mentioned record is the first one in Serbia as a psyllid predator. In Switzerland, it was registered by Wyniger and Burckhardt (2003) in galls of Psyllopsis fraxini. According to available literature data, $A$. amplicollis has not been published as a predator of P. buxi.

\section{2) Anthocoris confusus Reuter, 1884}

Trophic status. Zoophagous.

Distribution. Palaearctic.

Prey. Psylla buxi, from Buxus semprevirens, Sokobanja, 25.IX.2009, reared 1 q.

Registered as a psyllid predator on conifers (Wyniger and Burckhardt 2003) and aphids (Herard 1986). In the present paper, reported for the first time as a psyllid predator in Serbia. 


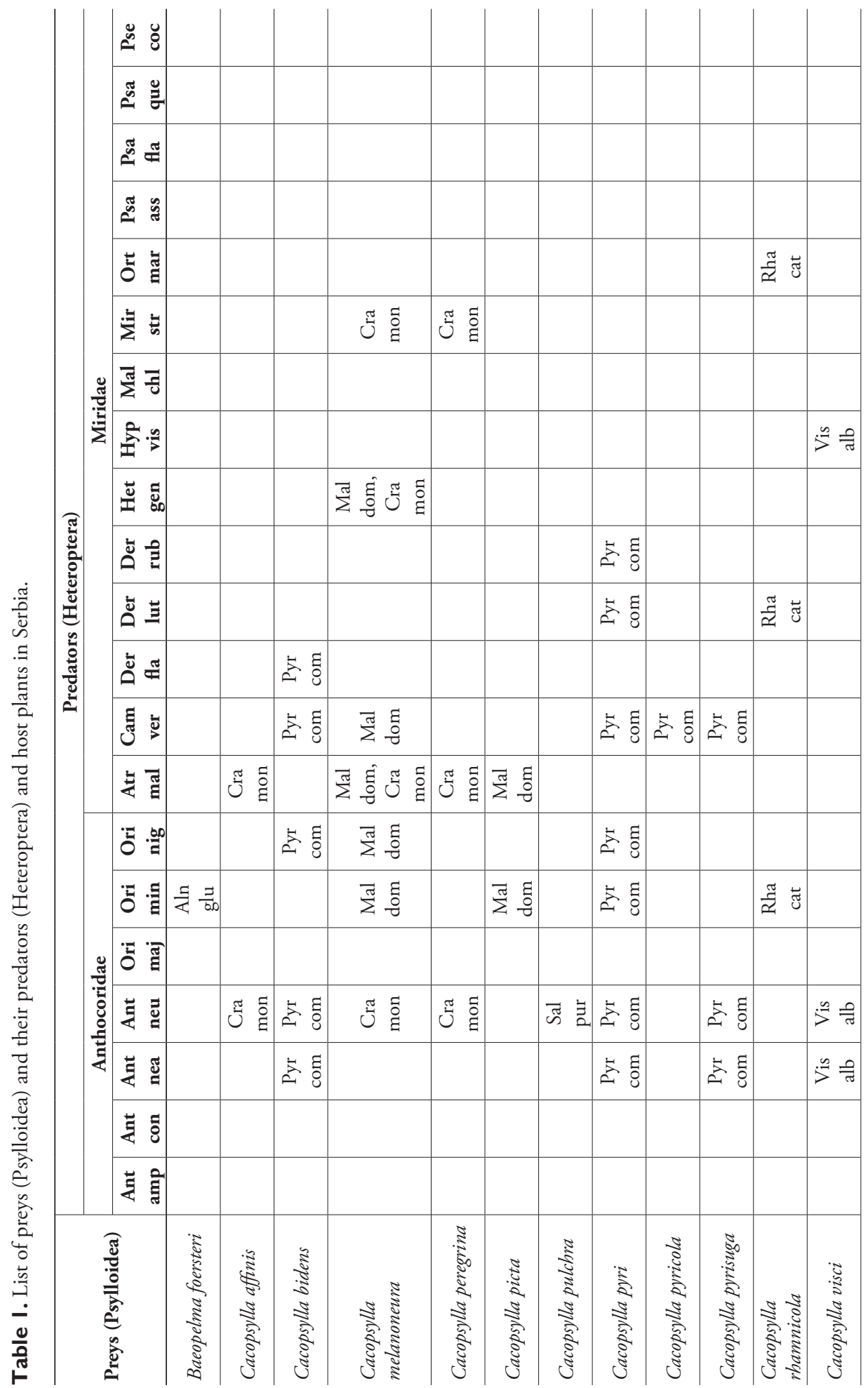




\begin{tabular}{|c|c|c|c|c|c|c|c|c|c|c|c|c|c|}
\hline$\stackrel{\circ}{\circ}$ & & & & & & & & ॠ & & & & & \\
\hline 哭 & & & & & & 芷言岀 के & & & 䍃 ㅇํㅇ & & & & \\
\hline$\cong$ & & & & & & 䍃 & & & & & & & \\
\hline 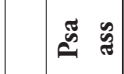 & & & & & & 舀 & & 釆 $\frac{\hat{b}}{\mathrm{w}}$ & & & & & \\
\hline 형 & & & & & & & & & & & & & \\
\hline$\frac{z}{2}$ & & & & & & & & & & & & & \\
\hline 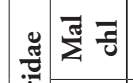 & & & 运 ซ & & & & & & & & & & \\
\hline$=\frac{2}{2}$ & & & & & & & & & & & & & \\
\hline 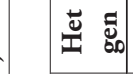 & & & & & & & & & & & & & \\
\hline$\stackrel{\text { ڤ̆ }}{\Xi}$ & & & & & & & & & & & & & \\
\hline 咅 & $\approx \cong .00$ & & & & & & & 岽哭 & & & & & 娄定 \\
\hline ڤ̆ Æ & & & 误 छ & & & 䍃 哭 & & & 䍃器 & & & & \\
\hline 馬 & & & & & & & & & & & & & \\
\hline 专嘾 & & & & & & & & & & & & & \\
\hline 50 & & 安咅 & & $\leqq \equiv$ & & & & 㐁 & & & & $\frac{\pi}{\tilde{\varkappa}} \overrightarrow{\tilde{\Xi}}$ & 是焉 \\
\hline ¿ & $\frac{0.00}{a}$ & & 误 छ & & & 䍃 & & 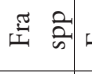 & 䍃 & & 䍃 & & \\
\hline 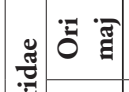 & & & & & & 䍃 & & & 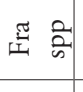 & & & & \\
\hline 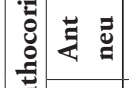 & & & & & 賹咢 & & & 番跑 & & & & & \\
\hline 莫焉 & $\stackrel{20.00}{\varrho}$ & & & & & 䍃 & & 䒻 के & 䍃 官 & ॠ्य के & 䍃 & & \\
\hline 莫营 & & & & & 着㤩 & & & & & & & & \\
\hline 莫竞 & & & & & 窇 & & & & & & & & \\
\hline 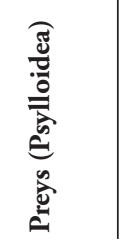 & 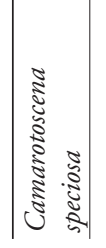 & 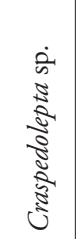 & 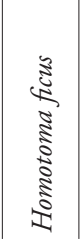 & 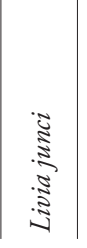 & 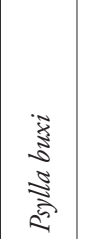 & 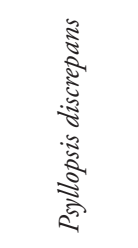 & 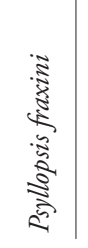 & 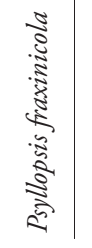 & 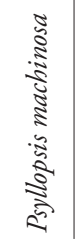 & 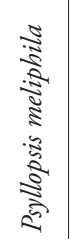 & 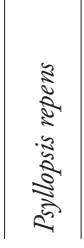 & 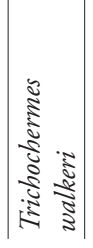 & 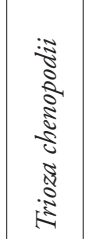 \\
\hline
\end{tabular}




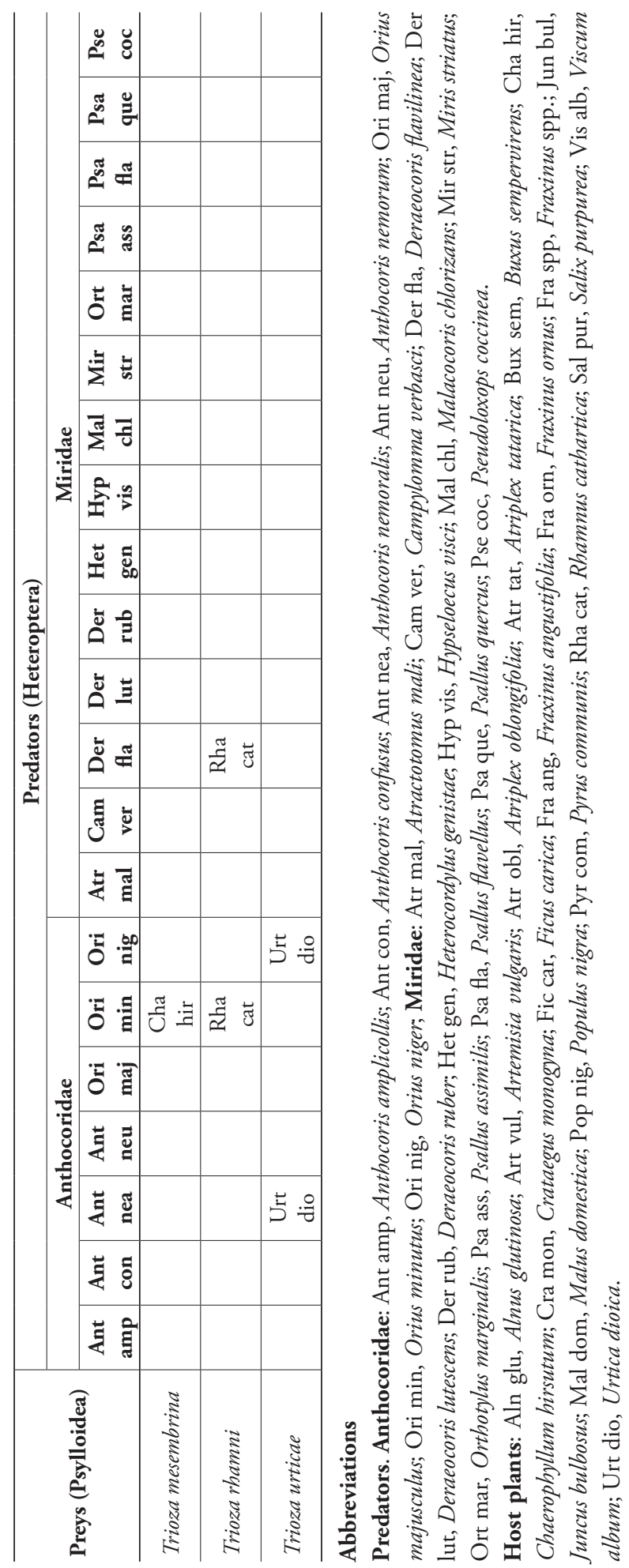




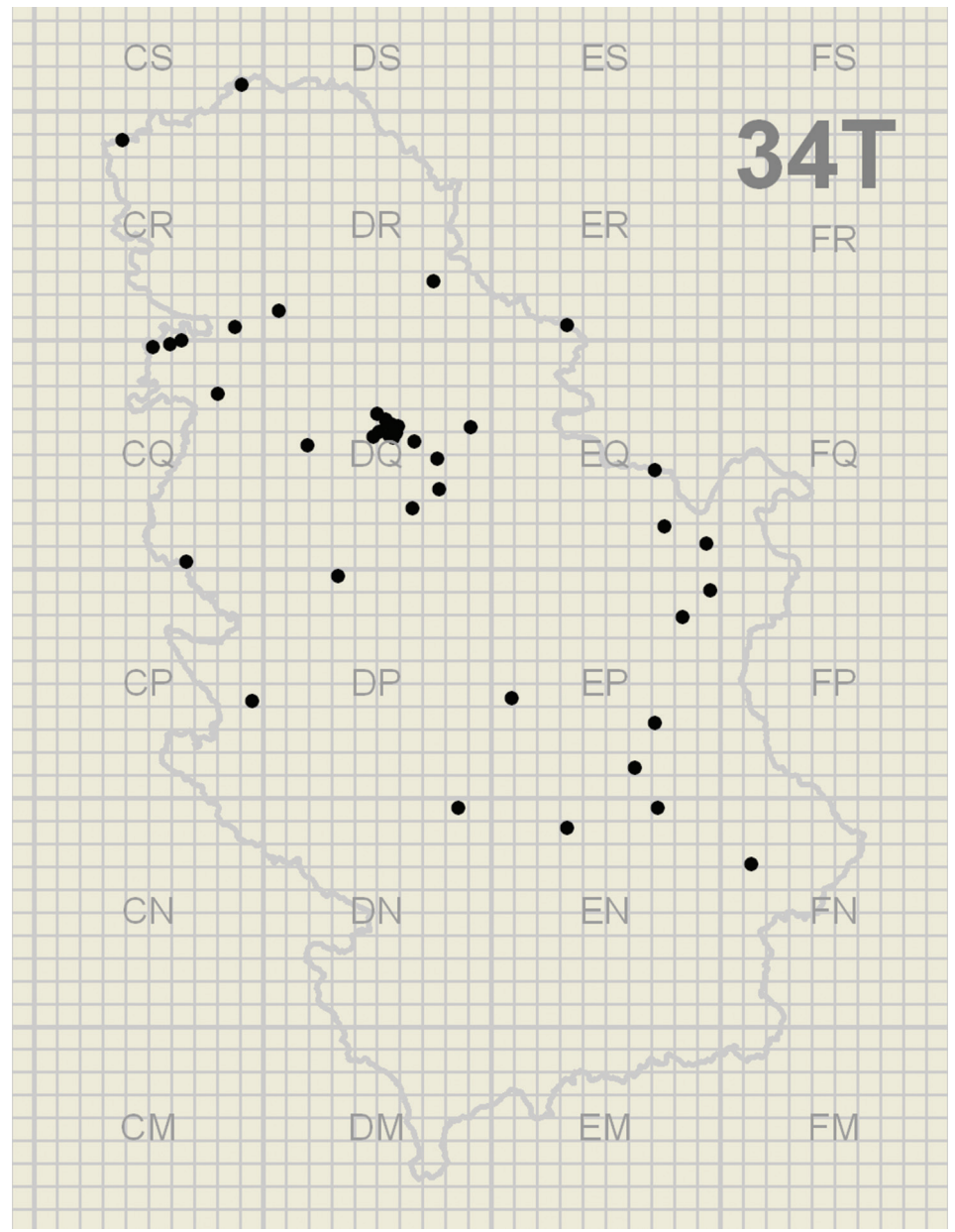

Figure I. Localities in Serbia where true bug predators of psyllids were collected.

\section{3) Anthocoris nemoralis (Fabricius, 1794)}

Trophic status. Zoophagous.

Distribution. Euro-Mediterranean.

Preys. Cacopsylla bidens (Šulc, 1907), from Pyrus communis, Beograd-Karaburma, 19.V.2006, reared 2 우 'ex larva'25.V.2006. Cacopsylla pyri, from Pyrus communis, Nemenikuće, 15.VI.2006, reared 10 'ex larva'22.VI.2006; Radmilovac, 2.IX.2005, reared 10; 12.VI.2006, reared $1 \delta^{\lambda}$ 'ex larva'25.VI.2006; 26.X.2006, collected 1§; 7.VI.2007, reared 1ð, 19 'ex larva'11.VI.2007, 20.VI.2007, collected 1 larva and 1 $\delta^{\lambda}$; 26.X.2008, reared 1 $\delta^{\lambda}$ 'ex larva'3.XI.2008. Cacopsylla pyrisuga, from Pyrus communis, Zemunski kej, 28.V.2007, collected 1ㅇ. Cacopsylla visci (Curtis, 1835), from Viscum album, Beograd-Bulevar Aleksandra Karađorđevića,

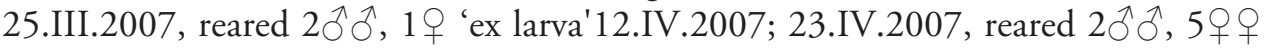


'ex larva'3.V.2007. Camarotoscena speciosa (Flor, 1861), from Populus nigra, Radmilovac, 22.V.2000, reared 10 'ex larva'25.V.2000; Zemun-Nova Galenika, 25.VIII.2008, collected 19 ; 1.IX.2008, reared 390 'ex larva'9.IX.2008; 10.IX.2008,

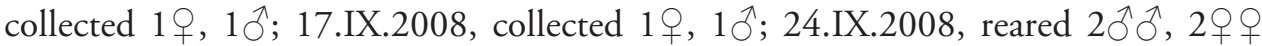
'ex larva'1.X.2008; 27.VII.2010, collected 10ิ, 1우, 18.X.2010, collected 10, 1 우. Psyllopsis discrepans (Flor, 1861), from Fraxinus sp., Beograd-Autokomanda,

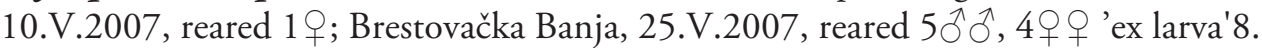

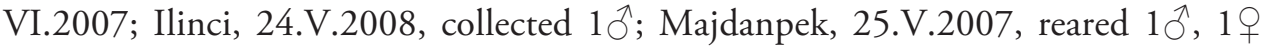
'ex larva'3.VI.2007, Milošev konak, 21.VI.2007, 1 đ. Psyllopsis fraxini, from Fraxinus sp., Veliko Središte, 30.V.2006, reared 2우. Psyllopsis fraxinicola (Foerster, 1848), from Fraxinus sp., Beograd-Autokomanda, 10.V.2007, reared 19; BeogradKalemegdan, 24.V.2007, collected 10خ; Brestovačka Banja, 25.V.2007, reared 5 $\hat{\jmath}$, 490 'ex larva' 8.VI.2007; Majdanpek, 25.V.2007, reared 10, 1 ㅇ 'ex larva'3.VI.2007; Veliko Središte, 4.VI.2006, reared 2 $9+$ 'ex larva'19.VI.2006. Psyllopsis machinosa Loginova, 1963, from Fraxinus sp., Beograd-Autokomanda, 10.V. 2007, reared 1․ Psyllopsis meliphila Löw, 1881, from Fraxinus sp., Nemenikuće, 15.VI.2006, collected $10^{\top}$. Psyllopsis repens Loginova, 1963, from Fraxinus sp., Beograd-Autokomanda, 14.X.2008, reared 19. Trioza urticae (Linnaeus, 1758), from Urtica dioica, Ilinci, 24.VI.2007, collected 2 우 0 .

A. nemoralis is an important component of the natural enemy community in pear and apple orchards where it provides biological control against arthropod pests, particularly psyllids (Horton et al. 2004). Investigating the predator-prey complex of $C$. pyri in a pear orchard in France, Herard (1986) found that A. nemoralis was the most efficient enemy against this pest. $A$. nemoralis is mentioned in many papers as a permanent member of biocomplexes of pear psyllids in Europe (Wheeler 2000b, Erler 2004, Sigsgaard et al. 2006). In Turkey, A. nemoralis was an equally present and efficient predator of pear psyllid C. pyri, both in treated and untreated orchards, but still insufficient for its full control (Erler 2004). In Spain, Jauset et al. (2006) determined $A$. nemoralis as a very efficient predator of $C$. pyri, both in treated and untreated pear orchards. Now, there is a mass production of $A$. nemoralis in companies specialized for biological control of harmful insects (Sigsgaard et al. 2006). The same authors reported that $A$. nemoralis mostly prefers $C$. pyri to aphids, and that it prefers laying eggs on pear to apple. $A$. nemoralis is a polyphagous predatory species having psyllids as a usual prey.

It is distributed in Europe and the Mediterranean. From Europe it was introduced into North America in 1963 in order to control pear psyllid C. pyricola, giving satisfactory results (Horton et al. 2004). This species has adapted to this region so well that it has suppressed autochthonous predatory species $A$. antevolens and A. melanocerus (Herard 1986, Horton et al. 2004). In Serbia, in a pear orchard, Pavićević (1977), Grbić et al. (1989) and Jerinić-Prodanović (2010) note a permanent presence of $A$. nemoralis, both during vegetation and winter period together with an overwintering adult of C. pyri. A. nemoralis was also reported as a predator of Psyllopsis repens in Serbia (Malenovský and Jerinić-Prodanović 2011). 
Table 2. Geographical coordinates of inspected localities.

\begin{tabular}{|c|c|c|c|c|}
\hline No & Locality & Latitude & Longitude & Altitude \\
\hline 1 & Bački breg & $45^{\circ} 55^{\prime} 21^{\prime \prime} \mathrm{N}$ & $18^{\circ} 55^{\prime} 24^{\prime \prime E}$ & 90 \\
\hline 2 & Bavanište & $44^{\circ} 48^{\prime} 42^{\prime \prime} \mathrm{N}$ & $20^{\circ} 53^{\prime} 10^{\prime \prime} \mathrm{E}$ & 80 \\
\hline 3 & Beloljin & $43^{\circ} 14^{\prime} 03^{\prime \prime} \mathrm{N}$ & $21^{\circ} 24^{\prime} 26^{\prime \prime} \mathrm{E}$ & 290 \\
\hline 4 & Beograd-Autokomanda & $44^{\circ} 47^{\prime} 20^{\prime \prime} \mathrm{N}$ & $20^{\circ} 28^{\prime} 20^{\prime \prime} \mathrm{E}$ & 100 \\
\hline 5 & Beograd-Banjica & $44^{\circ} 45^{\prime} 18^{\prime \prime} \mathrm{N}$ & $20^{\circ} 28^{\prime} 58^{\prime \prime} \mathrm{E}$ & 190 \\
\hline 6 & Beograd-Block 45 & $44^{\circ} 47^{\prime} 36^{\prime \prime} \mathrm{N}$ & $20^{\circ} 22^{\prime} 47^{\prime \prime} \mathrm{E}$ & 75 \\
\hline 7 & Beograd-Bulevar Aleksandra Karadjordjevića & $44^{\circ} 46^{\prime} 50^{\prime \prime} \mathrm{N}$ & $20^{\circ} 27^{\prime} 31^{\prime \prime E}$ & 175 \\
\hline 8 & Beograd-Hotel Jugoslavija & $44^{\circ} 49^{\prime} 36^{\prime \prime} \mathrm{N}$ & $20^{\circ} 25^{\prime} 22^{\prime \prime} \mathrm{E}$ & 75 \\
\hline 9 & Beograd-Hram Svetog Save & $44^{\circ} 47^{\prime} 53^{\prime \prime} \mathrm{N}$ & $20^{\circ} 28^{\prime} 03^{\prime \prime} \mathrm{E}$ & 120 \\
\hline 10 & Beograd-Kalemegdan & $44^{\circ} 49^{\prime} 19^{\prime \prime} \mathrm{N}$ & $20^{\circ} 26^{\prime} 52^{\prime \prime} \mathrm{E}$ & 110 \\
\hline 11 & Beograd-Karaburma & $44^{\circ} 48^{\prime} 48^{\prime \prime} \mathrm{N}$ & $20^{\circ} 29^{\prime} 15^{\prime \prime E}$ & 110 \\
\hline 12 & Beograd-Milošev konak & $44^{\circ} 46^{\prime} 38^{\prime \prime N} \mathrm{~N}$ & $20^{\circ} 25^{\prime} 36^{\prime \prime E}$ & 80 \\
\hline 13 & Beograd-Voždovac & $44^{\circ} 47^{\prime} 17^{\prime \prime} \mathrm{N}$ & $20^{\circ} 28^{\prime} 28^{\prime \prime} \mathrm{E}$ & 85 \\
\hline 14 & Brestovačka Banja & $44^{\circ} 03^{\prime} 36^{\prime \prime} \mathrm{N}$ & $22^{\circ} 02^{\prime} 36^{\prime \prime E}$ & 360 \\
\hline 15 & Dobra & $44^{\circ} 38^{\prime} 23^{\prime \prime N}$ & $21^{\circ} 54^{\prime} 06^{\prime \prime E}$ & 85 \\
\hline 16 & Draževac & $43^{\circ} 28^{\prime} 08^{\prime \prime} \mathrm{N}$ & $21^{\circ} 46^{\prime} 37^{\prime \prime} \mathrm{E}$ & 205 \\
\hline 17 & Galovica & $44^{\circ} 46^{\prime} 22^{\prime \prime} \mathrm{N}$ & $20^{\circ} 21^{\prime} 04^{\prime \prime E}$ & 75 \\
\hline 18 & Grocka & $44^{\circ} 41^{\prime} 21 " \mathrm{~N}$ & $20^{\circ} 42^{\prime} 02^{\prime \prime E}$ & 125 \\
\hline 19 & Ilinci & $45^{\circ} 06^{\prime} 41 " \mathrm{~N}$ & $19^{\circ} 07^{\prime} 16^{\prime \prime} \mathrm{E}$ & 80 \\
\hline 20 & Izvor & $43^{\circ} 04^{\prime} 58^{\prime \prime N} \mathrm{~N}$ & $22^{\circ} 23^{\prime} 57^{\prime \prime E}$ & 290 \\
\hline 21 & Kelebija & $46^{\circ} 08^{\prime} 59^{\prime \prime} \mathrm{N}$ & $19^{\circ} 35^{\prime} 10^{\prime \prime E}$ & 125 \\
\hline 22 & Kopaonik-Srebrenac & $43^{\circ} 19^{\prime} 02^{\prime \prime} \mathrm{N}$ & $20^{\circ} 50^{\prime} 08^{\prime \prime} \mathrm{E}$ & 1740 \\
\hline 23 & Klokočevac & $44^{\circ} 20^{\prime} 53^{\prime \prime} \mathrm{N}$ & $22^{\circ} 10^{\prime} 45^{\prime \prime} \mathrm{E}$ & 140 \\
\hline 24 & Koruška & $45^{\circ} 11^{\prime} 46^{\prime \prime N}$ & $19^{\circ} 34^{\prime} 23^{\prime \prime E}$ & 110 \\
\hline 25 & Lipovača & $45^{\circ} 08^{\prime} 24 " \mathrm{~N}$ & $19^{\circ} 16^{\prime} 53^{\prime \prime} \mathrm{E}$ & 165 \\
\hline 26 & Luka & $44^{\circ} 09^{\prime} 46^{\prime \prime} \mathrm{N}$ & $22^{\circ} 11^{\prime} 56^{\prime \prime} \mathrm{E}$ & 340 \\
\hline 27 & Majdanpek & $44^{\circ} 25^{\prime} 10^{\prime \prime} \mathrm{N}$ & $21^{\circ} 57^{\prime} 10^{\prime \prime E}$ & 520 \\
\hline 28 & Nemenikuće & $44^{\circ} 29^{\prime} 38^{\prime \prime N}$ & $20^{\circ} 34^{\prime} 00^{\prime \prime E}$ & 280 \\
\hline 29 & Niš & $43^{\circ} 18^{\prime} 35^{\prime \prime} \mathrm{N}$ & $21^{\circ} 53^{\prime} 50^{\prime \prime} \mathrm{E}$ & 200 \\
\hline 30 & Novi Sad-Detelinara & $45^{\circ} 15^{\prime} 50 " \mathrm{~N}$ & $19^{\circ} 48^{\prime} 56^{\prime \prime} \mathrm{E}$ & 80 \\
\hline 31 & Obedska bara & $44^{\circ} 44^{\prime} 10^{\prime \prime} \mathrm{N}$ & $19^{\circ} 59^{\prime} 15^{\prime \prime} \mathrm{E}$ & 80 \\
\hline 32 & Oparić & $43^{\circ} 44^{\prime} 40 " \mathrm{~N}$ & $21^{\circ} 06^{\prime} 38^{\prime \prime} \mathrm{E}$ & 310 \\
\hline 33 & Radenković & $44^{\circ} 56^{\prime} 01 " \mathrm{~N}$ & $19^{\circ} 29^{\prime} 05^{\prime \prime} \mathrm{E}$ & 80 \\
\hline 34 & Radmilovac & $44^{\circ} 45^{\prime} 15^{\prime \prime} \mathrm{N}$ & $20^{\circ} 34^{\prime} 39^{\prime \prime} \mathrm{E}$ & 160 \\
\hline 35 & Sokobanja & $43^{\circ} 38^{\prime} 41 " \mathrm{~N}$ & $21^{\circ} 53^{\prime} 11^{\prime \prime E}$ & 350 \\
\hline 36 & Sutjeska & $45^{\circ} 23^{\prime} 02^{\prime \prime N}$ & $20^{\circ} 41^{\prime} 53^{\prime \prime} \mathrm{E}$ & 75 \\
\hline 37 & Šid & $45^{\circ} 07^{\prime} 31 " \mathrm{~N}$ & $19^{\circ} 12^{\prime} 58^{\prime \prime} \mathrm{E}$ & 105 \\
\hline 38 & Umčari & $44^{\circ} 34^{\prime} 10^{\prime \prime} \mathrm{N}$ & $20^{\circ} 43^{\prime} 00^{\prime \prime} \mathrm{E}$ & 160 \\
\hline 39 & Uzovnica & $44^{\circ} 16^{\prime} 12^{\prime \prime N}$ & $19^{\circ} 19^{\prime} 47^{\prime \prime E}$ & 170 \\
\hline 40 & Veliko Središte & $45^{\circ} 12^{\prime} 54 " \mathrm{~N}$ & $21^{\circ} 25^{\prime} 30^{\prime \prime} \mathrm{E}$ & 120 \\
\hline 41 & Vrujci & $44^{\circ} 13^{\prime} 26^{\prime \prime} \mathrm{N}$ & $20^{\circ} 09^{\prime} 53^{\prime \prime} \mathrm{E}$ & 170 \\
\hline 42 & Zemun-Nova Galenika & $44^{\circ} 51^{\prime} 41^{\prime \prime N}$ & $20^{\circ} 22^{\prime} 11 " \mathrm{E}$ & 90 \\
\hline 43 & Zemunski kej & $44^{\circ} 50^{\prime} 29^{\prime \prime} \mathrm{N}$ & $20^{\circ} 25^{\prime} 06^{\prime \prime} \mathrm{E}$ & 75 \\
\hline 44 & Zlatibor-Kraljevske Vode & $43^{\circ} 43 ' 39 " \mathrm{~N}$ & $19^{\circ} 42^{\prime} 06^{\prime \prime} \mathrm{E}$ & 950 \\
\hline
\end{tabular}




\section{4) Anthocoris nemorum (Linnaeus, 1761)}

Trophic status. Zoophagous.

Distribution. Eurosiberian.

Preys. Cacopsylla affinis (Löw, 1880), from Crataegus monogyna, Ilinci, 27.IV.2008, reared 10 'ex larva'14.V. 2008. Cacopsylla bidens, from Pyrus communis, Nemenikuće, 15.VI.2006, reared 19. Cacopsylla melanoneura (Foerster, 1848), from Crataegus monogyna, Ilinci, 27.IV.2008, reared 10 'ex larva'14.V.2008; Klokočevac, 10.V.2008, collected 1ㅇ. Cacopsylla peregrina (Foerster, 1848), from Crataegus monogyna, Ilinci, 27.IV.2008, reared 10 'ex larva'14.V.2008. Cacopsylla pulchra (Zetterstedt, 1838), from Salix purpurea, Zlatibor-Kraljevske Vode, 30.IV.2007, collected 1 ㅇ and 1 larva. Cacopsylla pyri, from Pyrus communis, Novi

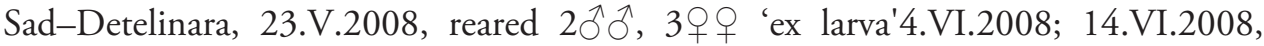
reared $3 \hat{\jmath}$ 'ex larva'17.VI.2008. Cacopsylla pyrisuga, from Pyrus communis, Grocka, 10.V.2008, reared 19 'ex larva'23.V.2008. Cacopsylla visci, from Viscum album, Beograd-Bulevar Aleksandra Karađorđevića, 23.IV.2007, reared 10 'ex larva'3.V.2007. Psylla buxi, from Buxus sempervirens, Vrujci, 1.VI.2009, collected

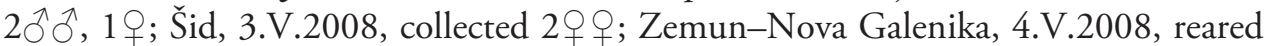

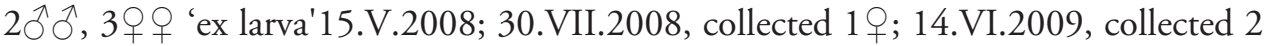
specimens. Psyllopsis fraxinicola, from Fraxinus angustifolia, Beograd-Hram Svetog Save, 14.IV.2008, reared $1 \delta^{\lambda}$ and 1 larva.

A. nemorum is noted as a predator of many insect species, in the first place Hemiptera, Diptera, eggs of Lepidoptera and mites (Herard 1986, Wheeler 2000b, Sigsgaard et al. 2006), already registered as a predator of both C. pyri and Ps. fraxini (Herard 1986). It is also largely reported as an efficient predator of apple psyllid Cacopsylla mali in Norway (Jonsson 1983). In England, Hodkinson and Flint (1971) determined A. nemorum as a predator of Psyllopsis fraxini collected from ash, while in Germany Novak and Achtziger (1995) registered it as a predator of hawthorn psyllids Cacopsylla melanoneura and $C$. peregrina. Sigsgaard et al. (2006) note $A$. nemorum as a more polyphagous species than $A$. nemoralis. They also determined in experimental conditions that $A$. nemorum prefers aphids to psyllids, and has a preference for laying eggs on apple rather than on pear.

A. nemorum is an Eurosiberian species, introduced to North America in order to control C. pyricola just like A. nemoralis, but without satisfactory results (Herard 1986).

A. nemorum is reported here for the first time as a predator of psyllids in Serbia.

\section{5) Orius (Heterorius) majusculus Reuter, 1884}

Trophic status. Zoophagous.

Distribution. Euro-Atlantic.

Preys. Psyllopsis discrepans and Psyllopsis machinosa, from Fraxinus spp., Beograd-Autokomanda, 6.V.2009, collected 10. 
O. majusculus was registered as a predator of psyllids (Herard 1986). It is noted as a predator of aphids, such as Diuraphis noxia and Schizaphis graminum in Russia, mites in France, whiteflies in greenhouses in Italy and pear psyllid Cacopsylla pyri in France (Péricart 1972, Herard 1986).

The present paper reports $O$. majusculus as a psyllid predator for the first time in Serbia and Psyllopsis discrepans and Ps. machinosa for the first time as a prey of O. majusculus.

\section{6) Orius (Heterorius) minutus (Linnaeus, 1758)}

Fig. 2

Trophic status. Zoophagous.

Distribution. Palaearctic.

Preys. Baeopelma foersteri (Flor, 1861), from Alnus glutinosa, Radenković, 3.VI.2006, collected 1q. Cacopsylla melanoneura, from Malus domestica, Beograd-Hotel Jugoslavija, 26.V.2005, reared 10̂; Ilinci, 2.V.2010, reared 10̂, 2 우 'ex larva'10.V.2010. Cacopsylla picta (Foerster, 1848), from Malus domestica, BeogradHotel Jugoslavija, 26.V.2005, reared 10. Cacopsylla pyri, from Pyrus communis, Radmilovac, 10.VII.2006, reared 10, 2 \% 'ex larva'20.VII.2006; 26.VII.2006, collected 10ิ; 4.IX.2006, collected 10ิ. Cacopsylla rhamnicola (Scott, 1876), from Rhamnus cathartica, Kelebija, 25.V.2005, reared 1 ㅇ 'ex larva'6.VI.2005. Camarotoscena speciosa, from Populus nigra, Zemun-Nova Galenika, 1.IX.2008, reared 10 'ex larva'9. IX.2008; 24.IX.2008, reared 10 'ex larva'1.X.2008. Homotoma ficus (Linnaeus,

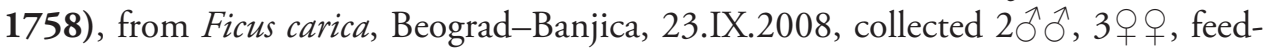
ing on eggs. Psyllopsis discrepans, from Fraxinus ornus, Ilinci, 21.V.2005, collected 10. Psyllopsis discrepans and Psyllopsis repens, from Fraxinus ornus, Beograd-Autokomanda, 7.IX.2008, reared 5 $\hat{\jmath}$, 4 우 'ex larva'11.IX.2008; 21.IX.2008, col-

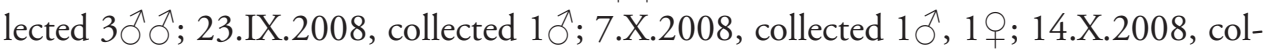
lected 1; 21.X.2008, collected 1 9 . Psyllopsis fraxinicola, from Fraxinus sp., Vrujci, 1.VII.2009, 1 9 . Psyllopsis machinosa, from Fraxinus ornus, Beograd-Autokomanda, 6.V.2009, reared 1 q 'ex larva'10.V.2009. Psyllopsis repens, from Fraxinus ornus, Beograd-Autokomanda, 21.VIII.2010, reared 19. Trioza mesembrina Burckhardt, 1986, from Chaerophyllum hirsutum, Kopaonik-Srebrenac, 7.VIII.2008, reared 10 . Trioza rhamni (Schrank, 1801), from Rhamnus cathartica, Kelebija, 25.V.2005, reared 1ㅇ 'ex larva'6.VI.2005; Ilinci, 2.V.2009, 10․

O. minutus is an extremely polyphagous species distributed in Europe, Siberia, China and Mediterranean region. Many authors determined it as a predator of harmful insect species from a number of orders (Thysanoptera, Diptera, Lepidoptera, Coleoptera and Hemiptera Homoptera). Already reported as a psyllid predator (Herard 1986). In France, Herard (1986) determined O. minutus as a predator of pear psyllids, primarily C. pyri, and hawthorn psyllids. Also in Slovenia, Vrabl and Matis (1977) register it as a predator of C. pyri and C. pyrisuga. In Serbia, Pavićević (1977) and Jerinić-Prodanović et al. (2010) determined O. minutus as a predator of $C$. pyri in 


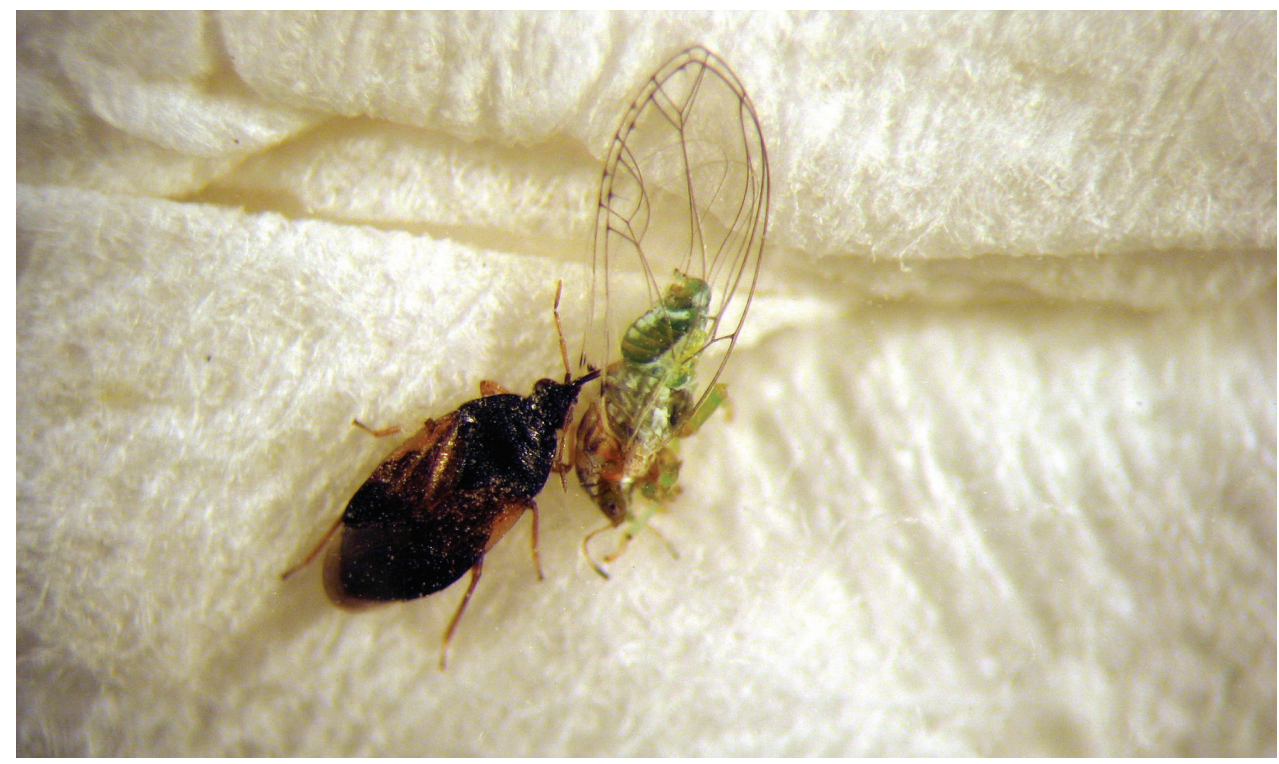

Figure 2. Orius minutus feeding on Trioza rhamni.

pear orchards. Malenovský and Jerinić-Prodanović (2011) also found it as a predator of Ps. repens. In Croatia, Arčanin and Balarin (1972) recognized the significance of $O$. minutus in the reduction of the mite Panonychus ulmi.

\section{7) Orius (Orius) niger Wolff, 1811}

Trophic status. Zoophagous.

Distribution. Palaearctic.

Preys. Cacopsylla bidens, from Pyrus communis, Ilinci, 13.X.2008, collected 10ิ. Cacopsylla melanoneura, from Malus domestica, Lipovača, 29.IV.2006, collected 10. Cacopsylla pyri, from Pyrus communis, Radmilovac, 26.VI.2006, reared 10 'ex larva'30.VI.2006; 10.VII.2006, reared 19 'ex larva'20.VII.2006. Craspedolepta sp., from Artemisia vulgaris, Sutjeska, 2.X.2009, reared $10^{\lambda}$ and 4 우. Psyllopsis fraxinicola, from Fraxinus ornus, Vrujci, 30.VI.2009, collected 1 specimen. Livia junci (Schrank, 1789), from Juncus bulbosus, Beograd-Block 45, 10.VIII.2005, reared $1{ }^{\lambda}$ 'ex larva'16.VIII.2005. Trichochermes walkeri (Foerster, 1848), from Rhamnus ca-

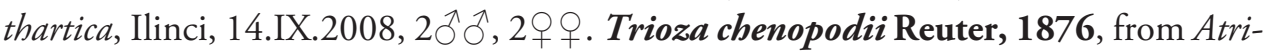
plex tatarica, Ilinci, 20.VIII.2006, $10^{\lambda}$ 'ex larva'23.VIII.2006. Trioza urticae, from Urtica dioica, Bački breg, 7.VI.2005, reared 10; Ilinci, 14.V.2005, reared 10, 1 우 'ex larva'27.V.2005; Luka, 25.V.2007, reared 10.

$O$. niger is widespread in Western Palaearctic, very rare in the Mediterranean region, also reported from China. It is a very polyphagous species, preying on aphids, psyllids, whiteflies, thrips, larvae of noctuids, mites (Péricart 1972, Herard 1986, Protić 1993). 
In south France, Herard (1986) determined Orius niger on pears as a predator of Cacopsylla pyri, but also collected it in a large number from Trioza urticae from nettle, which was surrounding the pear orchards. In Croatia, O. niger was determined along with O. minutus on Panonychus ulmi in an apple orchard (Arčanin and Balarin 1972).

In Serbia, Grbić et al. (1989), investigating pear psyllid predators, reported Orius spp., so we are not able to compare our results with theirs. In the same paper, authors mentioned the presence of other Orius species frequently during summer and autumn which is in accordance with our investigations.

\section{Miridae}

\section{8) Atractotomus mali Meyer-Dür, 1843}

Trophic status. Phytozoophagous.

Distribution. Palaearctic.

Preys. Cacopsylla affinis, from Crataegus monogyna, Koruška, 1.V.2008, reared 1 ㅇ 'ex larva'8.V.2008. Cacopsylla melanoneura, from Crataegus monogyna, Beograd-

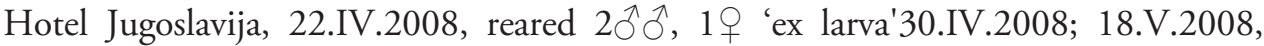

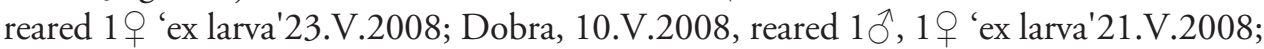
Draževac, 20.IV.2008, reared 1§, 19 2.V.2008; Koruška, 1.V.2008, reared 1 ㅇ 'ex larva'8.V.2008. Cacopsylla melanoneura, from Malus domestica, Ilinci, 24.V.2008, reared 1 ㅇ 'ex larva'30.V.2008; Oparić, 4.V.2008, reared 20' 'ex larva'19.V.2008; Ilinci, 2.V.2010, reared 1․ Cacopsylla peregrina, from Crataegus monogyna, Beograd-Hotel Jugoslavija, 22.IV.2008, reared 20 $\hat{\partial}$, 1 우 'ex larva'30.IV.2008; Dobra, 10.V.2008, reared 10, 1 ' 'ex larva'21.V.2008; Koruška, 1.V.2008, reared 1q 'ex larva' 8.V.2008. Cacopsylla picta, from Malus domestica, Ilinci, 24.V.2008, 1 ㅇ 'ex larva'30.V.2008.

A. mali has been reported so far as a predator of mites, aphids, thrips, psyllids, butterfly larvae and pupae (Wheeler 2000b). It was registered as a predator of apple psyllid Cacopsylla mali in Norway (Jonsson 1983) and of pear psyllid C. pyri in Greece (Santas 1987). In Germany, Novak and Achtziger (1995) registered it as a predator of hawthorn psyllids Cacopsylla spp.

First record of $A$. mali as a predator of $C$. picta. The above mentioned data are the first ones for $A$. mali as a psyllid predator in Serbia.

\section{9) Campylomma verbasci (Meyer-Dür, 1843)}

Trophic status. Zoophytophagous.

Distribution. Holarctic.

Preys. Cacopsylla bidens, from Pyrus communis, Ilinci, 24.V.2008, reared $10^{\lambda}$ 'ex larva'29.V.2008; Bavanište, 25.V.2006, reared 10, 1 ㅇ 'ex larva'30.V.2006. Cacopsylla pyri, Cacopsylla pyricola and Cacopsylla pyrisuga, from Pyrus communis, 
Bavanište, 25.V.2006, 1ठ, 1 ㅇ 'ex larva'30.V. 2006. Cacopsylla melanoneura, from Malus domestica, Ilinci, 2.V.2010, reared 20̂̉.

C. verbasci is a zoophytophagous species preying on apple aphids, pear psyllids, codling moth, thrips and mites (Wheeler 2000b). Its most common prey among insects are Aphis pomi and Cacopsylla mali, and among mites Panonychus ulmi and Tetranychus urticae (Hagen et al. 1999, Wheeler 2000b, Bradley 2007).

However, if there is a lack of prey, it can feed on apple fruits, rarely pear, causing the harm to their aesthetic value. Therefore, $C$. verbasci is a significant fruit pest in Canada (Hagen et al. 1999, Wheeler 2000a, Bradley 2007). Erler (2004) reported the presence of $C$. verbasci as a predator of $C$. pyri in treated and untreated pear orchards in Turkey, and Harizanova et al. (2012) mentioned it on Acizzia jamatonica in Bulgaria.

Already known in Serbia (Protić 1993) but in our investigations registered for the first time as a predator of psyllids in this country.

\section{0) Deraeocoris (Deraeocoris) flavilinea (A. Costa, 1862)}

Trophic status. Zoophytophagous.

Distribution. Western and Central Europe.

Preys. Cacopsylla bidens, from Pyrus communis, Beograd-Karaburma, 4.V.2006, reared 1 ㅇ 'ex larva'18.V.2006. Homotoma ficus, from Ficus carica, Beograd-Banjica,

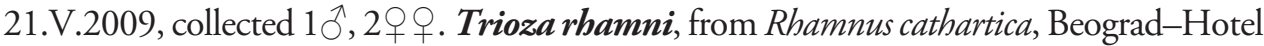
Jugoslavija, 26.V.2005, reared 10خ 'ex larva'29.V.2005. Psyllopsis discrepans and Psyllopsis machinosa, from Fraxinus angustifolia, Beograd-Autokomanda, 21.V.2009, $10^{\lambda}, 1$ \% .

D. flavilinea is reported so far as a predator of psyllids (Jerinić-Prodanović and Protić 2011, Simov et al. 2012). Until 1980's, it was known only from Sicily, from where it has spread to Central Europe where it is now considered as an invasive species (Rabitsch 2008). As a predator of psyllids, it has been known in Serbia since 2011 (Jerinić-Prodanović and Protić 2011).

\section{1) Deraeocoris (Deraeocoris) ruber (Linnaeus, 1758)}

Trophic status. Zoophytophagous.

Distribution. Holarctic.

Prey. Cacopsylla pyri, from Pyrus communis, Radmilovac, 10.VII.2006, reared 19.

A very polyphagous zoophytophagous species. A Holarctic species occurring in large quantities in the south of Europe.

Already mentioned as a predator of C. pyri (Herard 1986). It also preys on younger caterpillar instars of some butterflies, mites and various other small insects in apple orchards, on Rubus spp. and Urtica spp. as well as on aphids on Corylus spp. (Herard 1986).

Reported as a predator of $A$. jamatonica (Harizanova et al. 2012) in Bulgaria and C. pyri in Serbia (Jerinić-Prodanović et al. 2010). 


\section{2) Deraeocoris (Knightocapsus) lutescens (Schilling, 1836)}

Trophic status. Zoophagous.

Distribution. Euro-Mediterranean.

Preys. Cacopsylla pyri, from Pyrus communis, Izvor, 14.IV.2009, 10․ Cacopsylla rhamnicola, from Rhamnus cathartica, Obedska bara, 4.VI.2005, reared $10^{\text {t ' }} \mathrm{ex}$ larva'18.VI.2005. Camarotoscena speciosa, from Populus nigra, Zemun-Nova Galenika, 18.X.2010, collected 1․ Psyllopsis fraxinicola, from Fraxinus angustifolia, Zemun-Nova Galenika, 1.IX.2006, collected 19. Trioza chenopodii, from Atriplex

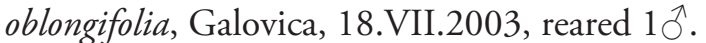

D. lutescens is a Mediterranean species, distributed also in small numbers in Central Europe. Known mainly as an egg predator of pear psyllid C. pyri and hawthorn psyllid C. crataegi (Herard 1986). It is also reported as a predator of aphids and mite Panonychus ulmi in apple orchards in Croatia (Arčanin and Balarin 1972) and in pear orchards as a predator of C. pyri in France and Turkey (Herard 1986, Erler 2004).

D. lutescens has been already registered in Serbia (Protić 1993) but here is reported for the first time as a predator of psyllids in this country.

\section{3) Heterocordylus (Heterodactylus) genistae (Scopoli, 1763)}

Trophic status. Phytozoophagous.

Distribution. Europe.

Prey. Cacopsylla melanoneura, from Malus domestica, Beloljin, 4.V.2008, collected 10; Ilinci, 20.V.2006, reared 10 'ex larva'25.V.2006; Uzovnica, 29.IV.2007, collected 1 specimen.

H. genistae is mentioned in the literature as a beneficial insect being a predator of psyllids both in larval and adult stage. It is registered as a predator of various other insects (Protić 1993, 1998).

In the present paper, we report $H$. genistae for the first time as a predator of psyllids in Serbia.

\section{4) Hypseloecus visci (Puton, 1888)}

Trophic status. Zoophagous.

Distribution. Europe.

Prey. Cacopsylla visci, from Viscum album, Beograd-Bulevar Aleksandra Karađorđevića, 25.III.2007, reared 4 우 우 'ex larva'16.IV.2007.

An exclusively zoophagous species.

Already known from Serbia as a psyllid predator (Jerinić-Prodanović and Protić 2011). 


\section{5) Malacocoris chlorizans Panzer, 1794}

Trophic status. Zoophagous.

Distribution. Eurasia.

Prey. Homotoma ficus, from Ficus carica, Beograd-Hotel Jugoslavija, 16.V.2007,

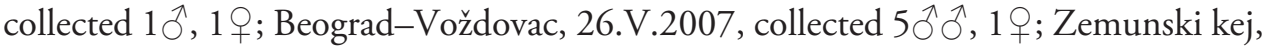
15.V.2008, collected 1 larva.

A general predator on aphids, psyllids, eggs and larvae of leaf miner moths (Wheeler 2000b, Wyniger and Burckhardt 2003). In Croatia, it is registered as a predator of Panonychus ulmi in apple orchards by Arčanin and Balarin (1972).

Malacocoris chlorizans has been already registered in Serbia (Protić 1998) but in the present paper is reported for the first time as a predator of psyllids in this country.

16) Miris striatus (Linnaeus, 1758)

Trophic status. Zoophagous.

Distribution. Europe, Central Asia.

Preys. Cacopsylla melanoneura and Cacopsylla peregrina, from Crataegus monogyna, Dobra, 10.V.2008, reared 1 + 'ex larva'21.V.2008.

Already reported from Serbia (Protić 1993, 1998). The above mentioned record is the first one of M. striatus as a predator of psyllids.

17) Orthotylus (Orthotylus) marginalis Reuter, 1884

Trophic status. Zoophytophagous.

Distribution. Eurosiberian.

Prey. Cacopsylla rhamnicola, from Rhamnus cathartica, Beograd-Hotel Jugoslavija, 15.V.2008, reared 1 ㅇ 'ex larva'19.V.2008.

O. marginalis is registered as a predator of aphids and psyllids (Wheeler 2000b). In Finland and Russia, it was mentioned as a predator of C. mali (Jonsson 1983).

Registered in Serbia (Protić 2011) but here reported for the first time as a psyllid predator.

18) Psallus (Hylopsallus) assimilis Stichel, 1956

Trophic status. Phytozoophagous.

Distribution. Europe.

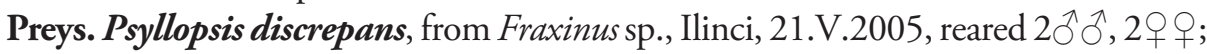
Umčari, 25.V.2007, reared 1․ Psyllopsis fraxinicola and Psyllopsis discrepans, from Fraxinus sp., Beograd-Autokomanda, 10.V.2007, reared 5 $\widehat{\partial}$, 8 우 0 'ex larva'16.V.2007. 


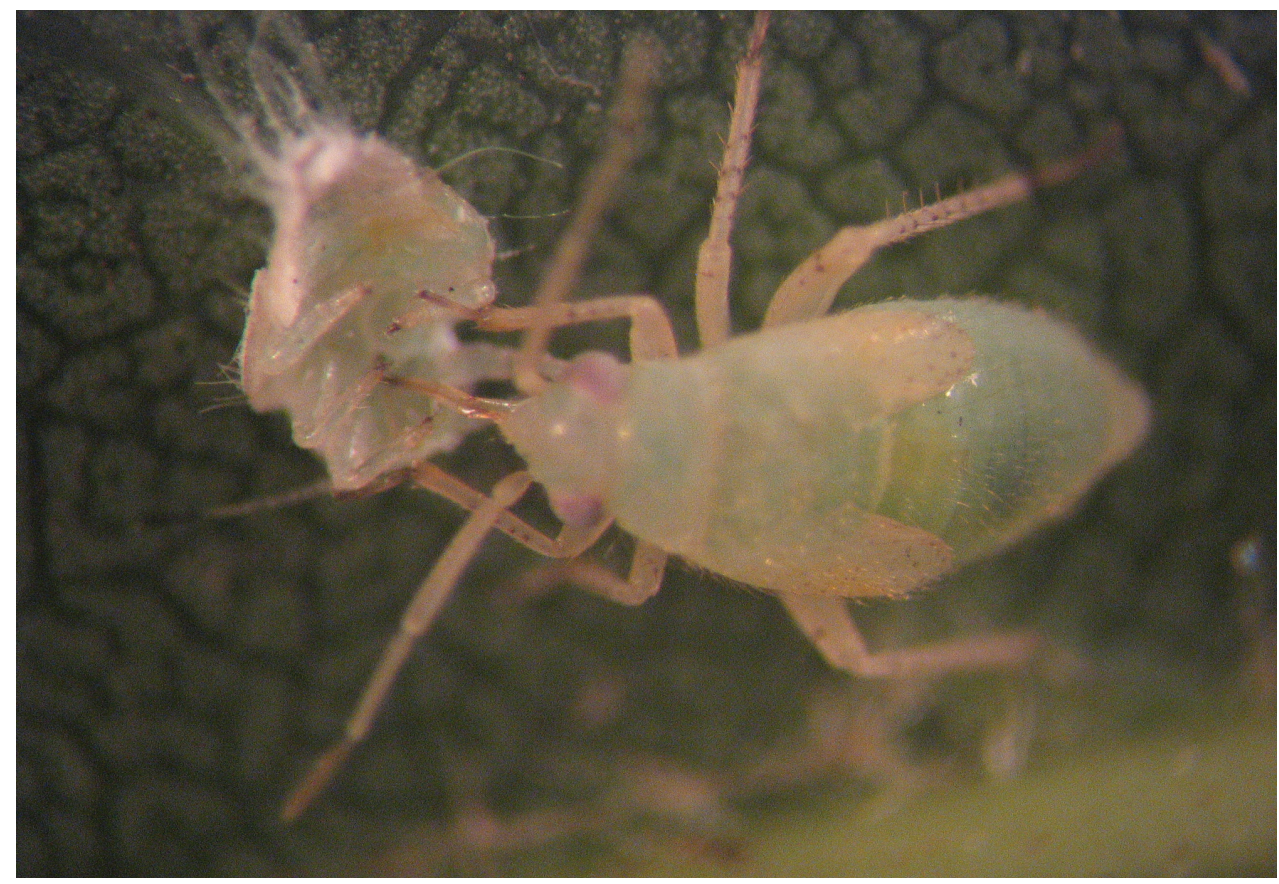

Figure 3. Larva of Psallus flavellus feeding on Psyllopsis fraxinicola.

Already known as a predator of various insect species, inlcuding psyllids.

Previously registered in Serbia (Protić 1998) but in the present paper reported for the first time as a psyllid predator in this country.

19) Psallus (Psallus) flavellus Stichel, 1933

Fig. 3

Trophic status. Phytozoophagous.

Distribution. Europe.

Preys. Psyllopsis spp., from Fraxinus sp., Beograd-Autokomanda, 8.V.2010, reared $3 \hat{\jmath} \widehat{\jmath}, 1$.. Psyllopsis discrepans, from Fraxinus sp., Beograd-Autokomanda, 13.V.2010, reared $2 \widehat{\jmath}, 1$.

Previously registered in Serbia (Protić 2011). Reported here for the first time as a psyllid predator.

20) Psallus (Phylidea) quercus Kirschbaum, 1856

Trophic status. Phytozoophagous.

Distribution. Europe, Asia. 
Preys. Psyllopsis discrepans, from Fraxinus ornus, Ilinci, 24.V. 2008, collected 1우 17.V. 2009, 10, 2 우. Psyllopsis discrepans and Ps. machinosa, from Fraxinus sp., Beograd-Autokomanda, 6.V.2009, reared 4 우 'ex larva'10.V.2009; 21.V.2009, reared $1 \hat{0}, 3$ 우.

So far known as a predator of aphids, psyllids, thrips, spiders and eggs of various insects (Protić 1998).

Registered in Serbia (Protić 2011) but here reported for the first time as a psyllid predator in this country.

\section{1) Pseudoloxops coccinea Meyer-Dür, 1843}

Trophic status. Zoophagous.

Distribution. Euro-Mediterranean.

Preys. Psyllopsis fraxinicola, from Fraxinus sp., Niš, 27.V.2008, collected 10ิ. Psyllopsis sp., Fraxinus sp., Beograd-Autokomanda 8.V.2010, reared $10^{\Uparrow}$ 'ex larva'21.V.2010.

Registered in Serbia (Protić 1999). Reported for the first time as a predator of psyllids.

\section{Conclusions}

From 28 psyllid species and 19 host plants, we reared or collected 21 species of true bugs belonging to the families Anthocoridae and Miridae. According to available literature data, 12 of the recorded species are zoophagous, while the other nine have mixed nutrition.

Miris striatus, Pseudoloxops coccinea and Psallus flavellus (Miridae) have not been registered as psyllid predators so far. Sixteen species of true bugs are recorded here for the first time as psyllid predators in Serbia (Anthocoris amplicollis, A. nemorum, A. confusus, Orius majusculus, O. niger, Atractotomus mali, Campylomma verbasci, Deraeocoris lutescens, Heterocordylus genistae, Malacocoris chlorizans, Orthotylus marginalis, Psallus assimilis, Ps. quercus, Ps. flavellus, Miris striatus and Pseudoloxops coccinea).

From the family Anthocoridae, we identified seven species: Anthocoris amplicollis, $A$. confusus, $A$. nemoralis, A. nemorum, Orius majusculus, $O$. minutus and $O$. niger. The most polyphagous among them was $O$. minutus, found on 13 species of psyllids: Baeopelma foersteri, Cacopsylla melanoneura, C. picta, C. pyri, C. rhamnicola, Camarotoscena speciosa, Homotoma ficus, Psyllopsis discrepans, Ps. fraxinicola, Ps. machinosa, Ps. repens, Trioza mesembrina and T. rhamni.

From the family Miridae, we reared or collected 14 species: Atractotomus mali, Campylomma verbasci, Deraeocoris flavilinea, D. ruber, D. lutescens, Heterocordylus genistae, Hypseloecus visci, Malacocoris chlorizans, Miris striatus, Orthotylus marginalis, Psallus assimilis, P. flavellus, P. quercus and Pseudoloxops coccinea. Among them, the most polyphagous were C. verbasci, D. flavillinea and D. lutescens, each registered on five psyllid species. 
Most of predatory true bugs are registered on deciduous perennial plants. We found the highest number of predatory true bugs on psyllids which overwinter on host plant and have more than one generation per year, e.g. Cacopsylla pyri, Psyllopsis fraxinicola and $P$ s. discrepans. On each of them, seven predatory true bugs were registered. Species from the genus Psallus were registered as predators only of psyllid genus Psyllopsis.

Further investigations are necessary for the preservation of known beneficial predatory true bugs and finding of new ones, potentially usable for biological control on economically significant psyllid species.

\section{Acknowledgments}

We thank Alexi Popov and two anonymous reviewers for comments and suggestions on the manuscript. They helped us improve our manuscript. We owe special thanks also to Goran Prodanović and Dragica Smiljanić, who collected a part of insect material.

\section{References}

Arčanin B, Balarin I (1972) Predatorske vrste Heteroptera zastupljene u fauni jabučnih nasada Hrvatske. Acta entomologica Jugoslavica 8 (1/2): 11-21.

Bradley SH (2007) Campylomma verbasci (Meyer) (Hemiptera: Miridae). Mullein plant bug. Biological control. A guide to Natural Enemies in North America. http://www.nysaes. cornell.edu/ent/biocontrol/

Erler F (2004) Natural enemies of the pear psylla Cacopsylla pyri in treated vs untreated pear orchards in Antalya, Turkey. Phytoparasitica 32 (3): 295-304. doi: 10.1007/BF02979824 Grbić M, Lakić B, Mihajlović Lj (1989) Predators and parasitoids of Psylla pyri L. (Hom. Psyllidae) in Vojvodina (YU). OILB Workshop Lutte integree en verger de poires, 28 juin-1 juillet 1988. Changius (Rac) Switzerland. Proceedings, 1-11.

Hagen KS, Mills NJ, Gordh G, Mcmurty JA (1999) Terestrial arthropod predators of insects and mite pests. In: Bellows TS, Fisher TW (Eds) Principles and Applications of Biological Control. Handbook of Biological Control. Academic Press, San Diego, 383-461. doi: 10.1016/B978-012257305-7/50063-1

Harizanova V, Stoeva A, Mohamedova M (2012) Preliminary study on the invasive Acizzia jamatonica (Hemiptera: Psyllidae) and its predators in Bulgaria. Agricultural Science and Technology 4 (1): 56-61.

Herard F (1985) Analysis of parasite and predator populations observed in pear orchards infested by Psylla pyri (L.) (Hom. Psyllidae) in France. Agronomie 5: 773-778. doi: 10.1051/ agro: 19850901

Herard F (1986) Annotated list of the entomophagous complex associated with pear psylla, Psylla pyri (L.) (Hom. Psyllidae) in France. Agronomie 6: 1-34. doi: 10.1051/agro:19860101

Hodkinson ID, Flint PWH (1971) Some predators from the galls of Psyllopsis fraxini L. (Hem., Psyllidae). Entomologist's monthly magazine 107: 11-12. 
Horton D, Lewis TM, Broers DA (2004) Ecological and geographic range expansion of the introduced predator Anthocoris nemoralis (Heteroptera: Anthocoridae) in North America: Potential for nontarget effects? American entomologist 50 (1): 18-30.

Jauset AM, Artigues M, Sarasúa MJ (2006) Abundance and seasonal distribution of natural enemies in treated vs untreated pear orchards in Lleida (NE Spain). International Organization for Biological and Integrated Control of Noxious Animals and Plants. West Palearctic Regional Section. Workshop on Arthropod Pest Problems in Pome Fruit Production at Lleida (Spain), 4-6 September 2006. Book of abstracts, 54.

Jerinić-Prodanović D (2010) Diverzitet lisnih buva (Homoptera: Psylloidea) i njihovih prirodnih neprijatelja u Srbiji, sa posebnim osvrtom na vrste značajne u poljoprivredi. PhD thesis. Belgrade, Serbia: University of Belgrade, Faculty of Agriculture, 339 pp. doi: 10.2298/ PIF1001029J

Jerinić-Prodanović D, Protić Lj, Mihajlović Lj (2010) Predatori i parazitoidi Cacopsylla pyri (L.) (Hemiptera: Psyllidae) u Srbiji. Pesticidi i Fitomedicina (Belgrade) 25 (1): 29-42. doi: 10.2298/PIF1001029J

Jerinić-Prodanović D, Protić Lj (2011) New data on true bug predators (Heteroptera: Miridae) of jumping plant-lice (Sternorrhyncha: Psylloidea) in Serbia. Acta entomologica Serbica $16(1 / 2): 143-146$.

Jonsson N (1983) The life history of Psylla mali Schmidberger (Hom., Psyllidae); and its relationship to the development of the apple blossom. Fauna Norvegica. Norvegian Journal of Entomology, Ser. B, 30 (1): 3-8.

Kerzhner IM, Josifov M (1999) Miridae Hahn, 1833. In: Aukema B, Rieger Ch (Eds) Catalogue of the Heteroptera of the Palaearctic Region. Vol. 3. Cimicomorpha II. The Netherlands Entomological Society, Amsterdam, 577 pp.

Luiz de Queiroz D, Burckhardt D, Majer J (2012) Integrated pest management of eucalypt psyllids (Insecta, Hemiptera, Psylloidea). In: Larramendy ML, Soloneski S (Eds) Integrated Pest Management and Pest Control - Current and Future Tactics. InTech, 385-412. http://www.intechopen.com/books/integrated-pest-management-and-pest-control-current-and-future-tactics/integrated-pest-management-of-eucalypt-psyllids-insecta-hemiptera-psylloidea- doi: 10.5772/32631

Malenovský I, Jerinić-Prodanović D (2011) A revised description of Psyllopsis repens Loginova, 1963 (Hemiptera: Psylloidea: Psyllidae), with first records from Europe. Archives of Biological Sciences (Belgrade) 63 (1): 257-286.

Novak H, Achtziger R (1995) Influence of heteropteran predators (Het., Anthocoridae, Miridae) on larval populations of hawthorn psyllids (Hom., Psyllidae). Journal of Applied Entomology 119: 479-486. doi: 10.1111/j.1439-0418.1995.tb01321.x

Pavićević B (1977) Morfologija i biologija vrsta roda Psylla (Psyllidae, Homoptera) na kruškama u Srbiji. PhD thesis. Belgrade, Serbia: University of Belgrade, Faculty of Agriculture, 139 pp.

Péricart J (1972) Hémiptères Anthocoridae, Cimicidae, Microphysidae de l'Ouest Paléarctique. In: Faune de l'Europe et du bassin méditerranéen 7. Masson et Cie, Paris, 403 pp.

Protić Lj (1993) Proučavanje faune stenica (Heteroptera) u voćnim zasadima Srbije. PhD thesis. Belgrade, Serbia: University of Belgrade, Faculty of Agriculture, 294 pp. 
Protić Lj (1998) Catalogue of the Heteroptera fauna of Yugoslav countries. Part one. Natural History Museum, Belgrade. Special issue 38: 1-215.

Protić Lj (1999) Six species of Miridae new to the fauna of Yugoslavia. Acta entomologica Slovenica 7 (1): 53-57.

Protić Lj (2011) Heteroptera. Natural History Museum, Belgrade, Posebna izdanja 43: 259 pp. Protić Lj, Stojanović A (2003) New records of Anthocoris amplicollis Horváth (Heteroptera: Anthocoridae) on the Balkan peninsula. Acta entomologica Serbica, 8 (1/2): 103-104.

Rabitsch W (2008) Alien true bugs of Europe (Insecta: Hemiptera: Heteroptera). Zootaxa 1827: 1-44.

Santas LA (1987) The predators'complex of pear-feeding psyllids in unsprayed wild pear trees in Greece. Entomophaga 32 (3): 291-297. doi: 10.1007/BF02373253

Sigsgaard L, Esbjerg P, Philipsen H (2006) Controlling pear psyllids by mass-releasing Anthocoris nemoralis and A. nemorum (Heteroptera: Anthocoridae). Journal of Fruit and Ornamental Plant Research 14 (Suppl. 3): 89-98.

Simov N, Langourov M, Grozeva S, Gradinarov D (2012) New and interesting records of alien and native true bugs (Hemiptera: Heteroptera) from Bulgaria. Acta zoologica bulgarica 64 (3): 241-252.

Vrabl S, Matis G (1977) Prilog poznavanju biologije i suzbijanja kruškinih buva u Sloveniji. Zaštita bilja 28 (1), No. 139: 41-52.

Wagner E (1970-1971) Die Miridae Hahn, 1831 des Mittelmeerraumes und der Makaronesischen Inseln (Hemiptera, Heteroptera). Entomologische Abhandlungen, Suppl. 39: 421 pp.

Wagner E (1975) Die Miridae Hahn, 1831 des Mittelmeerraumes und der Makaronesischen Inseln (Hemiptera, Heteroptera). Entomologische Abhandlungen, Suppl. 40: 484 pp.

Wheeler Jr AG (2000a) Plant bugs (Miridae) as plant pests. In: Schaefer CW, Pannizzi AR (Eds) Heteroptera of Economic Importance. CRC Press, Washington, 37-87.

Wheeler Jr AG (2000b) Predacious plant bugs (Miridae). In: Schaefer CW, Pannizzi AR (Eds) Heteroptera of Economic Importance. CRC Press, Washington, 657-693.

Wyniger D, Burckhardt D (2003) Die Landwanzenfauna (Hemiptera, Heteroptera) von Basel (Schweiz) und Umgebung. Mitteilungen der Schweizerischen entomologischen Gesellschaft 76: 1-136. 